Y. C. Minh and E. F. van Dishoeck, eds.

\title{
Physical Properties of Molecular Envelopes in Low-Mass Star-Forming Regions
}

\author{
Nagayoshi Ohashi \\ Academia Sinica Institute of Astronomy \& Astrophysics, P.O. Box 1-87, \\ Nankang, Taipei 115, Taiwan
}

\begin{abstract}
We have carried out interferometric observations of preprotostellar and protostellar envelopes in Taurus. Protostellar envelopes are dense gaseous condensations with young stellar objects or protostars, while pre-protostellar envelopes are those without any known young stellar objects. Five pre-protostellar envelopes have been observed in CCS $J_{N}=3_{2}-2_{1}$, showing flattened and clumpy structures of the envelopes. The observed CCS spectra show moderately narrow line widths, $\sim 0.1$ to $\sim 0.35 \mathrm{~km} \mathrm{~s}^{-1}$. One pre-protostellar envelope, L1544, shows a remarkable velocity pattern, which can be explained in terms of infall and rotation. Our $\mathrm{C}^{18} \mathrm{O} J=1-0$ observations of 8 protostellar envelopes show that they have also flattened structures like pre-protostellar envelopes but no clumpy structures. Four out the eight envelopes show velocity patterns that can be explained by motions of infall (and rotation). Physical properties of pre-protostellar and protostellar envelopes are discussed in detail.
\end{abstract}

\section{Introduction}

Molecular envelopes are sites of star and planet formation. In order to understand how stars and planets form in molecular envelopes we need to investigate the physical properties of molecular envelopes. This is also very important for studying the chemical evolution of molecular envelopes because the physical conditions place crucial constrains on chemical models.

My task in this volume is to provide the physical properties of molecular envelopes. Particularly, I focus on the molecular envelopes in low-mass star forming regions, where stars are mainly formed in an isolated mode (e.g. Taurus), because their star formation processes are relatively simple, providing us with a basic picture of star formation. Since low-mass protostar candidates, deeply embedded in molecular envelopes, were discovered by IRAS (Beichman et al. 1986), these "protostellar envelopes" have been studied in detail. In addition, molecular envelopes without any detectable embedded sources are recently actively studied (e.g. Ward-Thompson et al. 1994; André et al. 1996). These envelopes, often called "pre-protostellar envelopes," or "starless dense cores," are considered to be in a very early stage of star formation, because they are dense enough to form stars although no detectable embedded sources form yet. 
In order to understand a scenario of star formation in molecular envelopes, it is necessary to study both protostellar and pre-protostellar envelopes.

Millimeter-wavelength interferometers providing high angular and velocity resolutions are the key to detailed studies of molecular envelopes. Interferometric observations at millimeter wavelengths, however, have been technically difficult for a long time, particularly for low-mass star forming regions, mainly because such instruments were not sensitive enough to detect weak emission from low-mass star forming regions: recent developments of low-noise receivers at millimeter-wavelengths enable us to make sensitive interferometric observations of molecular envelopes in low-mass star forming regions. In this paper, interferometric observations of pre-protostellar and protostellar envelopes in the Taurus low-mass star forming region are presented. Physical properties of the two types of molecular envelopes are discussed, with emphasis on their kinematical properties (see also Ohashi 1999).

\section{CCS Observations of Pre-protostellar Envelopes}

Pre-protostellar envelopes in the Taurus star-forming region were observed in CCS $J_{N}=3_{2}-2_{1}(\nu=33.751375 \mathrm{GHz})$ using the Berkeley-Illinois-Maryland Association (BIMA) array. CCS, one of the carbon-chain molecules, has the following characteristics: (1) optically thin, (2) an intrinsically narrow line width $(\sim 0.09$ $\mathrm{km} \mathrm{s}^{-1}$ at $T=10 \mathrm{~K}$ ), and (3) no hyperfine structures. The second and third characteristics are specifically important to investigating velocity fields of preprotostellar envelopes because they are often kinematically quiet and have narrow line widths. CCS was systematically surveyed toward both pre-protostellar and protostellar envelopes (Suzuki et al. 1992), demonstrating that CCS is more abundant in pre-protostellar envelopes. Hence, CCS is a good molecule to probe pre-protostellar envelopes. Only a few pre-protostellar envelopes, however, have been imaged in CCS (TMC1C: Hirahara et al. 1992, Langer et al. 1995; L1498: Kuiper et al. 1996).

The BIMA array is well-suited to mapping pre-protostellar envelopes. The first advantage is its wide field of view: the BIMA array provides a wide field of view of $\sim 5^{\prime}$ at $33 \mathrm{GHz}$. Since pre-protostellar envelopes tend to be moderately extended, the wide field of view is important to image pre-protostellar envelopes with less missing flux. The second advantage is its high velocity resolution: the frequency resolution was $\sim 6 \mathrm{kHz}$, providing a high velocity resolution of $\sim 0.054$ $\mathrm{km} \mathrm{s}^{-1}$ even at $33 \mathrm{GHz}$. Such a high velocity resolution is powerful to investigate the fine velocity structures of the envelopes. The typical angular resolution was $\sim 15^{\prime \prime}$, corresponding to $\sim 2000$ AU at the distance of the Taurus star-forming region $(d \sim 140 \mathrm{pc})$.

\subsection{Geometrical structures of CCS pre-protostellar envelopes}

Figure 1 shows the obtained CCS total intensity maps of the five pre-protostellar envelopes. For TMC1C, we observed three fields to cover the whole TMC1C cloud. It is remarkable that the observed CCS envelopes are elongated, presumably due to their flattened geometry viewed at high inclination to the line of sight. Protostellar envelopes also often show similar flattened structures (see $\S 3)$. These facts suggest that the flattened geometry of molecular envelopes has 


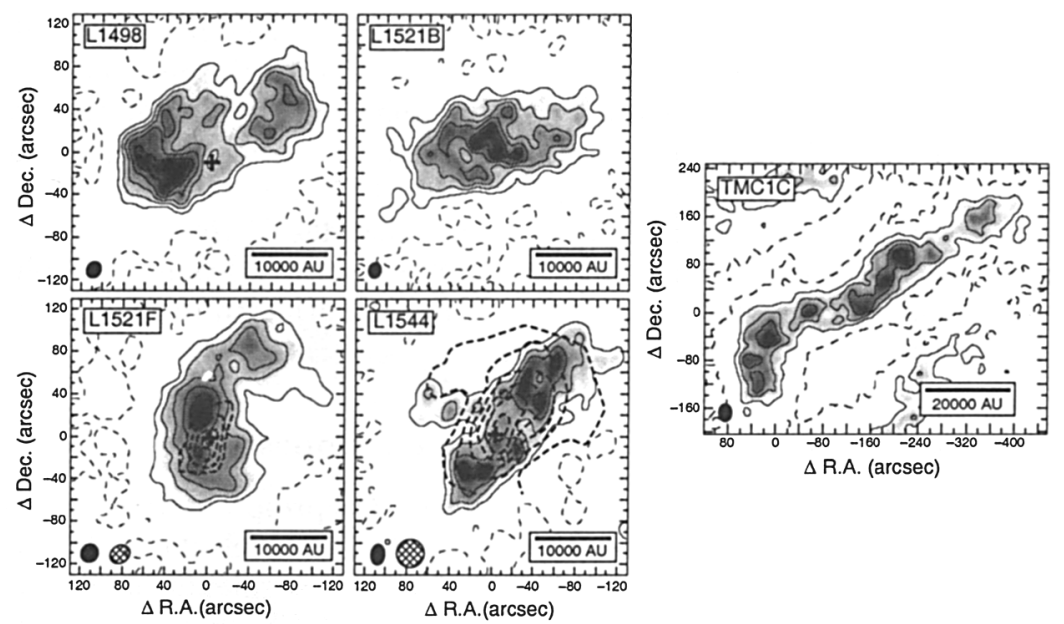

Figure 1. The CCS total intensity maps observed with the BIMA array are shown using thin contours with grey scale. The contour spacing is $2 \sigma$, starting at $\pm 2 \sigma$. Beam sizes of the CCS maps are shown by filled ellipses. For L1521F and L1544, $\mathrm{N}_{2} \mathrm{H}^{+}$maps are also shown by thick dashed contours, while peak positions of dust continuum emission are shown by crosses in the maps of L1498, L1521F, and L1544.

already formed before detectable embedded sources are formed in the envelopes. However, the size of the flattened structures are different between the two types of envelopes: the major axes of the flattened structures of the pre-protostellar envelopes are $\sim 10000$ AU scale, roughly 10 times larger than the typical size scale of the protostellar envelopes. We also note that the elongated structure of TMC1C is probably not only due to a flattened structure of the envelope but also due to its filamentary structure.

In Fig. 1, the position of the dust emission peaks are also plotted for three objects, L1498 (Willacy et al. 1998), L1521F (Moriarty-Schieve et al. 2000), and L1544 (Ward-Thompson et al. 1999). It is obvious that dust emission is stronger in the vicinity of the central regions of the envelopes, while CCS is stronger in the outer regions of the envelopes, showing a clear anticorrelation between the distributions of dust and CCS. Since some other molecules such as $\mathrm{N}_{2} \mathrm{H}^{+}$and CS show prominent peaks in the vicinity of the central regions [see contour maps of $\mathrm{N}_{2} \mathrm{H}^{+}$for L1521F (Ohashi et al. 2000) and L1544 (Tafalla et al. 1998) shown in Fig. 1; see also the CS map of L1498 (Kuiper et al. 1996)], dust emission basically traces the total amount of matter $\left(\mathrm{H}_{2}\right.$ and dust). Hence, the anticorrelation between CCS and dust suggests that CCS is heavily depleted in the inner regions of the envelopes. The relative abundance of the CCS molecule is considered to be decreased in the inner regions of pre-protostellar envelopes by photochemistry (Suzuki et al. 1992) and depletion onto grains (Bergin \& Langer 1997; see also Bergin, this volume). Such CCS depletion in the inner regions results in a ring-like structure of CCS envelopes. In fact, the appearances of the CCS envelopes of L1498 and L1544 are consistent with flattened, ring-like structures viewed at high inclination angles. 
Another remarkable geometrical feature of the CCS envelopes is their clumpy structure. Each map in Fig. 1 shows several peaks due to clumps. The clumpy structure is more obvious in channel velocity maps. The size of the clumps is typically $30^{\prime \prime}-40^{\prime \prime}$. It is important to note that the clumpy structure of the pre-protostellar envelopes is observed not only in CCS but also in $\mathrm{N}_{2} \mathrm{H}^{+}$. This may suggest that the clumpy structure is due to real density fluctuations rather than due to inhomogeneous molecular abundances caused by chemical evolution, because two molecules, which are different from each other in terms of chemical properties, show similar clumpy structures.

\subsection{Line widths of the CCS pre-stellar envelopes}

One remarkable kinematical characteristic of the CCS pre-protostellar envelopes is their narrow line width. In order to investigate the CCS line widths in the envelopes, each CCS image was divided into several segments having almost the same size as the beam size of the image, and CCS spectra averaged in each segment were obtained. The resulting CCS spectra were fitted assuming a Gaussian shape, showing that the line widths of the CCS spectra range in FWHM from $\sim 0.10$ to $\sim 0.35 \mathrm{~km} \mathrm{~s}^{-1}$. The measured line width, often called the total line width $\left(\Delta V_{\text {total }}\right)$, includes both the thermal line width $\left(\Delta V_{\text {thermal }}\right)$ due to thermal motion and the non-thermal line width $\left(\Delta V_{\text {nonthermal }}\right)$ due to nonthermal motion, i.e. $\Delta V_{\text {total }}^{2}=\Delta V_{\text {thermal }}^{2}+\Delta V_{\text {nonthermal }}^{2}$. The narrowest width is equivalent to the thermal line width of CCS at $10 \mathrm{~K}, \sim 0.09 \mathrm{~km} \mathrm{~s}^{-1}$. Note that some of the spectra obviously show two velocity components, which are due to (at least) two clumps coinciding along the same line of sight. Such spectra were fitted by two Gaussians. It must also be noted that the CCS spectra with wider line widths may have multiple velocity components due to multiple clumps along the same line of sight because those line profiles are not well fitted by a single Gaussian. If this is the case, then the actual line width of each velocity component is narrower than those derived from fittings with a single Gaussian.

Spatial distributions of $\Delta V_{\text {total }}$ give information on the spatial variations of non-thermal motions in molecular envelopes on the assumption that $\Delta V_{\text {thermal }}$ does not spatially vary (e.g. Fuller \& Myers 1992; Goodman et al. 1998). When we examined the spatial distribution of the CCS line width, it was found that there is no systematic variation, such that spectra with narrower line widths are located close to the central part of the envelope, although wider line widths tend to be observed from positions where the CCS total intensity is higher. This may suggest that there is not a systematic spatial variation of nonthermal motions in the CCS envelopes. It is, however, important to note that CCS may not trace well a spatial variation of the line width even if it exists, because CCS traces only the outer parts of the pre-protostellar envelopes, as was discussed in the previous section. If this is true, then it may be important to compare the line width of CCS with that of $\mathrm{N}_{2} \mathrm{H}^{+}$, which seems to trace the inner regions of pre-protostellar envelopes. Thus we compared line widths of CCS and $\mathrm{N}_{2} \mathrm{H}^{+}$ observed at the same line of sight in L1521F: interestingly, it was found that the total line width of CCS $\left(\sim 0.28 \mathrm{~km} \mathrm{~s}^{-1}\right)$ is larger than that of $\mathrm{N}_{2} \mathrm{H}^{+}(\sim 0.13$ $\mathrm{km} \mathrm{s}^{-1}$ ). This comparison may suggest that CCS shows a larger nonthermal velocity than $\mathrm{N}_{2} \mathrm{H}^{+}$because the thermal velocity of CCS is smaller than that of $\mathrm{N}_{2} \mathrm{H}^{+}$if the gas temperature is the same. This result could be explained by non- 

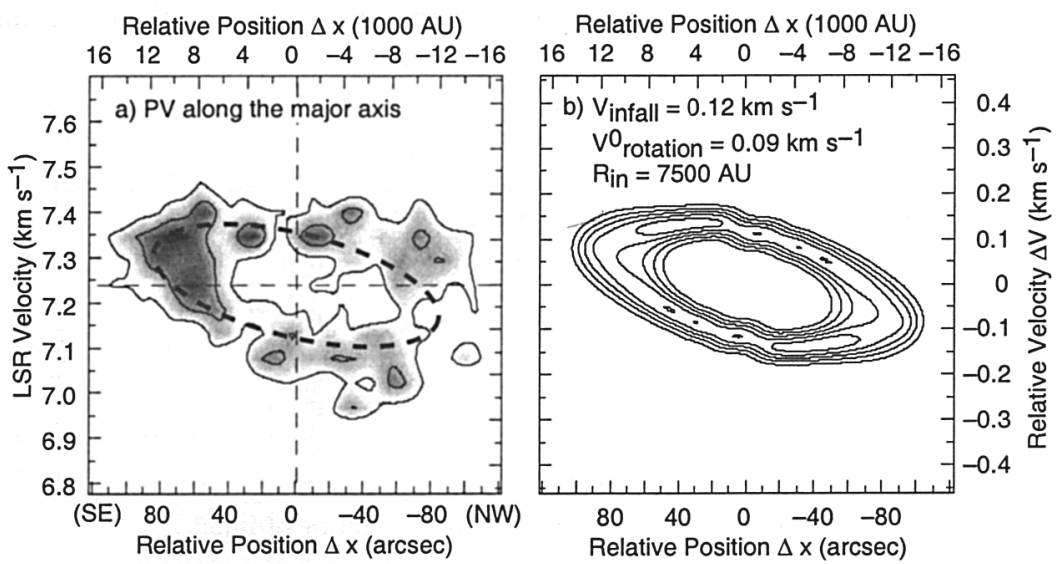

Figure 2. (a) Observed PV diagram cut along the projected major axis. (b) PV diagram calculated from a model envelope.

thermal motions, which become smaller in the inner regions of pre-protostellar envelopes. More systematic comparisons of the line width between CCS and $\mathrm{N}_{2} \mathrm{H}^{+}$may be important to discuss the spatial variation of nonthermal motions in pre-protostellar envelopes.

\subsection{Kinematic structure of CCS pre-protostellar envelopes: the case of $\mathbf{L 1 5 4 4}$}

All observed CCS pre-protostellar envelopes show detectable velocity gradients, ranging in magnitude from 1 to $8 \mathrm{~km} \mathrm{~s}^{-1} \mathrm{pc}^{-1}$. One of the envelopes, L1544, shows a particularly remarkable kinematical structure, which can be explained by infall and rotation as shown below.

In order to investigate the velocity structure of L1544 in detail, a positionvelocity (PV) diagram along the major axis is shown in Fig. 2a. Two remarkable features were found in the diagram:

1. There are two velocity components in the inner parts of the condensation $\left(-40^{\prime \prime} \leq \Delta x \leq+40^{\prime \prime}\right.$ in Fig. 2a), whereas these two components merge into a single velocity component at each of the southeastern and northwestern edges of the condensation. Since the two velocity components are observed at the same positions along the projected major axis, they are coincident along the line-of-sight.

2. There is a global velocity gradient from the southeast to the northwest (i.e. along the major axis).

Thus the whole velocity structure of the CCS condensation can be represented as a "tilted ellipse", as shown by the dashed curve in Fig. 2a. The elliptical velocity structure is roughly symmetrical with respect to $V_{\mathrm{LSR}} \sim 7.25 \mathrm{~km} \mathrm{~s}^{-1}$, suggesting that this velocity seems to be the systemic velocity of this system. This suggests that the two velocity components seen in Fig. 2a are actually blueshifted and redshifted parts of a single kinematical system. 
Table 1. Sources observed in the $\mathrm{C}^{18} \mathrm{O}$ mini survey and observational results.

\begin{tabular}{|c|c|c|c|c|c|c|c|c|c|}
\hline IRAS source & Name & $\begin{array}{c}L_{\text {bol }} \\
\left(\mathrm{L}_{\odot}\right)\end{array}$ & $\begin{array}{c}T_{\text {bolo }}{ }^{a} \\
(\mathrm{~K})\end{array}$ & Class & $\begin{array}{l}\text { Radius } \\
\text { (AU) }\end{array}$ & $\begin{array}{l}\text { Mass } \\
\left(\mathrm{M}_{\odot}\right)\end{array}$ & Infall $^{b}$ & Rotation $^{c}$ & Ref \\
\hline $04368+2557$ & L1527 IRS & 2.1 & 59 & 0 & 2000 & 0.04 & $\mathrm{Y}$ & $\mathrm{Y}$ & 1 \\
\hline $04289+1802$ & L1551NE & 6.0 & 75 & 0 & 1300 & 0.03 & $?$ & $\mathrm{Y}$ & 2 \\
\hline $04287+1801$ & L1551 IRS5 & 33 & 97 & I & 1200 & 0.06 & Y & $\mathrm{Y}$ & 3 \\
\hline $04361+2547$ & TMR1 & 2.8 & 144 & I & & & - & - & 4 \\
\hline $04169+2702$ & B213NW & 0.74 & 170 & I & 1100 & 0.02 & $?$ & $\mathrm{Y}$ & 5 \\
\hline $04365+2535$ & TMC1A & 2.2 & 172 & I & & & $(\mathrm{N})^{d}$ & $(\mathrm{Y})^{d}$ & 4,5 \\
\hline $04239+2436$ & & 1.3 & 236 & I & 1500 & 0.02 & $\mathrm{Y}$ & $?$ & 4 \\
\hline $04016+2610$ & L1489 IRS & 3.7 & 238 & I & 1000 & 0.008 & Y & Y & 4 \\
\hline
\end{tabular}

${ }^{a}$ Bolometric temperature (Chen et al. 1995).

${ }^{b}$ Whether or not the velocity gradient can be explained in terms of infall.

${ }^{c}$ Whether or not the velocity gradient can be explained in terms of rotation.

${ }^{d}$ The ${ }^{13} \mathrm{CO}$ observations show rotation but not infall (Ohashi et al. 1997b).

References.- (1) Ohashi et al. 1997a; (2) Momose 1998; (3) Momose et al. 1998; (4) Ohashi 1999; Ohashi et al. 1997b

The appearance of the L1544 CCS envelope is consistent with a flattened, ring-like structure viewed at a high inclination angle to the line of sight. Taking into account this geometrical structure, the two velocity components overlapping with each other along the line of sight can be explained by radial motion in the plane of the envelope, while the velocity gradient seen along the projected major axis may represent rotation of the CCS envelope. In fact, the observed PV diagram is reproduced by a simple model ring with infall and rigid rotation. In the model, $V_{\text {infall }}$ is constant throughout, while $V_{\text {rot }}$ increases as $V_{\text {rot }}=V_{\text {rot }}^{\mathrm{o}}\left(R / R_{\text {out }}\right)$, where $V_{\text {rot }}^{\mathrm{o}}$ is the rotation velocity at the outer radius of the envelope $R_{\text {out }}=15000$ AU. When $V_{\text {infall }}=0.12 \mathrm{~km} \mathrm{~s}^{-1}, V_{\mathrm{rot}}^{\mathrm{o}}=0.09 \mathrm{~km} \mathrm{~s}^{-1}$, and the inner radius of the envelope $R_{\text {in }}=7500 \mathrm{AU}$, the observed PV diagram along the major axis is well reproduced by the model, as shown in Fig. 2b. Inward motions in L1544 were also suggested from a spectroscopic method (Tafalla et al. 1998; Williams et al. 1999). Our result is remarkable since the observations resolve the velocity field and show direct evidence for infall motions in the pre-protostellar envelope L1544.

\section{3. $\mathrm{C}^{18} \mathrm{O}$ Mini Survey of Protostellar Envelopes}

Protostellar envelopes associated with embedded IRAS sources in Taurus were systematically observed using the Nobeyama Millimeter Array (NMA) and the Owens Valley Millimeter Array. Eight protostellar IRAS sources with $L_{b o l} \gtrsim 1$ $\mathrm{L}_{\odot}$, listed in Table 1, were selected for this mini survey. According to $T_{b o l}$ (Chen et al. 1995), two sources out of the eight are categorized into class 0 . The observations were done in $\mathrm{C}^{18} \mathrm{O} J=1-0$, which is most probably optically thin in protostellar envelopes. The typical angular resolution was $\sim 5^{\prime \prime}$, corresponding to $700 \mathrm{AU}$ at the distance of Taurus, while the velocity resolution was $\sim 0.2$ $\mathrm{km} \mathrm{s}^{-1}$. 

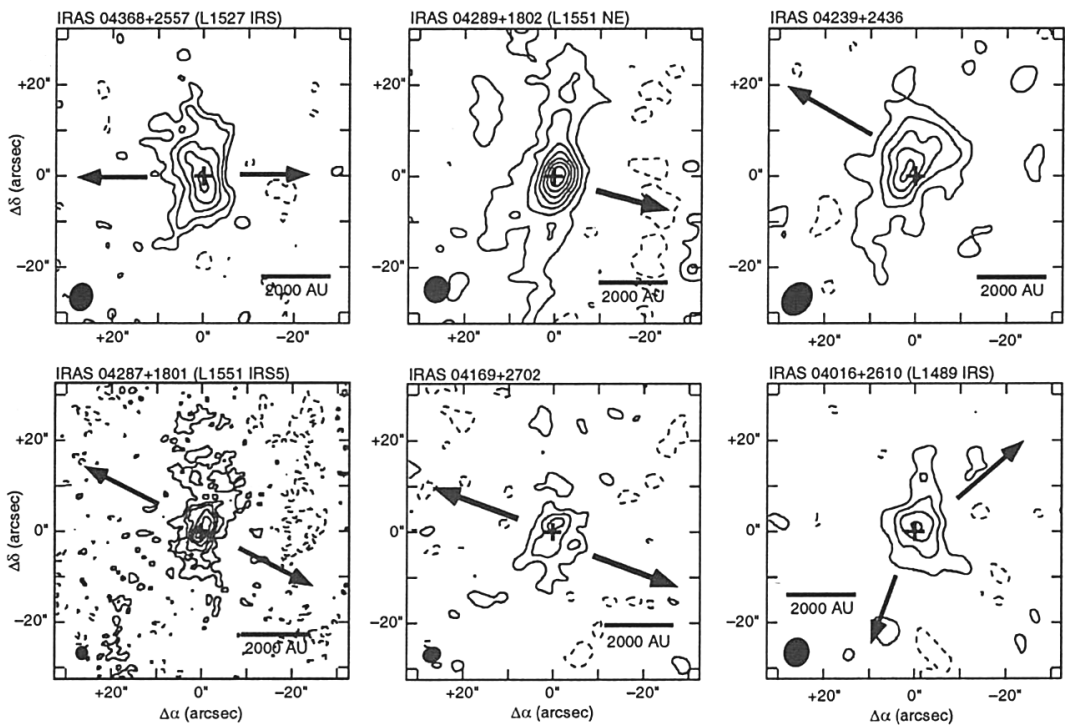

Figure 3. $\quad \mathrm{C}^{18} \mathrm{O}$ total intensity maps of protostellar envelopes in Taurus. The contour spacing is $2 \sigma$, starting at $\pm 2 \sigma$. Dashed lines show negative contours. Crosses show the positions of central protostellar sources while arrows show the directions of the associated outflows/jets.

\subsection{Physical properties of $\mathbf{C}^{18} \mathrm{O}$ envelopes}

Observational results are summarized in Table 1. Six sources, except for 04361+ 2547 and $04365+2535$, show detectable $\mathrm{C}^{18} \mathrm{O}$ emission. $\mathrm{C}^{18} \mathrm{O}$ total intensity maps of those 6 sources are presented in Figure 3. It is clear that each $\mathrm{C}^{18} \mathrm{O}$ condensation has a prominent peak close to the central source. It is also remarkable that every $\mathrm{C}^{18} \mathrm{O}$ envelope shows more or less an elongated structure, which is almost perpendicular to the direction of the associated outflow or jet. The ratio between the projected major and minor axes ranges from 2.1 to 3.1. This suggests that the $\mathrm{C}^{18} \mathrm{O}$ envelopes have flattened structures. We note, however, that flattened structures may be spatially thick. A good example is L1527 having an almost edge-on envelope: our $\mathrm{C}^{18} \mathrm{O}$ map shows that the envelope is spatially resolved along even the minor axis.

All $\mathrm{C}^{18} \mathrm{O}$ envelopes show clear velocity gradients. The velocity gradients are divided into three types: one along the major axis, one along the minor axis, and one along an intermediate direction between the major and minor axes. Infall yields a velocity gradient along the minor axis, while rotation yields a gradient along the major axis. When both infall and rotation exist in the envelope, we expect a velocity gradient along an intermediate direction between the major and minor axes (Hayashi et al. 1993; Momose et al. 1998). Taking into account these points, it is found that the detected velocity gradients can be explained by either infall, or rotation, or both. We summarized kinematical properties of the envelopes in the 6th and 7th columns of Table 1. 
Table 2. Physical properties of $\mathrm{C}^{18} \mathrm{O}$ infalling envelopes.

\begin{tabular}{lcccrrr}
\hline IRAS source & Class & $\begin{array}{c}V_{\text {infall }}{ }^{a} \\
\left(\mathrm{~km} \mathrm{~s}^{-1}\right)\end{array}$ & $\begin{array}{c}V_{\text {rot }}{ }^{a} \\
\left(\mathrm{~km} \mathrm{~s}^{-1}\right)\end{array}$ & $\begin{array}{c}\dot{M}^{b} \\
\left(\mathrm{M}_{\odot} \mathrm{yr}^{-1}\right)\end{array}$ & $\begin{array}{c}R_{\mathrm{c}}{ }^{c} \\
(\mathrm{AU})\end{array}$ & Ref \\
\hline $04368+2557$ & 0 & 0.3 & 0.05 & $1 \times 10^{-6}$ & 100 & 1 \\
$04287+1801$ & $\mathrm{I}$ & 0.5 & 0.2 & $5 \times 10^{-6}$ & 160 & 3 \\
$04239+2436$ & $\mathrm{I}$ & 0.4 & $<0.2$ & $1 \times 10^{-6}$ & $<100$ & 4 \\
$04016+2610$ & $\mathrm{I}$ & $1.1^{d}$ & $0.6^{d}$ & $2 \times 10^{-6}$ & 170 & 4 \\
\hline
\end{tabular}

${ }^{a} V_{\text {infall }}$ and $V_{\text {rot }}$ were measured at the same radius for each source.

${ }^{b}$ Mass infall rate, $\dot{M}$, was derived by $\dot{M}=M R / V_{\text {infall, }}$ where $M$ and $R$ are the mass and the radius of the envelope, respectively (see Table 1 ).

${ }^{c}$ Centrifugal radius $R_{\mathrm{c}}$, where $V_{\text {rot }}=V_{\text {infall }}$ was estimated from the measured $V_{\text {infall }}$ and $V_{\text {rot }}$ under the assumption that $V_{\text {infall }}$ increases as $R^{-0.5}$ and $V_{\text {rot }}$ increases as $R^{-1}$ where $R$ is the radius of the envelope.

${ }^{d}$ The systemic velocity of the source was assumed to be $\sim 7.7 \mathrm{~km} \mathrm{~s}^{-1}$.

References.- see Table 1

\subsection{Physical properties of $\mathbf{C}^{18} \mathrm{O}$ infalling envelopes}

In our mini survey, infalling motions were found around four sources. In this subsection, we discuss physical properties of these four infalling envelopes. Table 2 summarizes four important parameters of the infalling envelopes, i.e. infall velocity $V_{\text {infall }}$, rotation velocity $V_{\text {rot }}$, mass infall rate $\dot{M}$, and centrifugal radius $R_{\mathrm{c}}$. When we compare $V_{\text {infall }}$ and $V_{\text {rot }}$, it is found that $V_{\text {infall }}$ is 2-6 times larger than $V_{\text {rot }}$, suggesting that the envelopes are not rotationally supported but dynamically infalling. Another result suggestive of dynamical infall for the envelopes is the radial dependence of $V_{\text {infall }}$ and $V_{\text {rot }}$ : $V_{\text {infall }}$ increases as $R^{-0.5}$ while $V_{\text {rot }}$ increases as $R^{-1}$ (Momose et al. 1998).

It is found that every infalling envelope shows a similar $\dot{M},(1-6) \times 10^{-6}$ $\mathrm{M}_{\odot} \mathrm{yr}^{-1}$. An important fact is that the derived values are consistent with those expected from theory: according to the inside-out collapse model, the mass accretion rate is approximately estimated by $a^{3} / G$, where $a$ is the sound speed, and $G$ is the gravitational constant (Shu et al. 1987). When the cloud temperature is $\sim 10 \mathrm{~K}, \dot{M} \sim 2 \times 10^{-6} \mathrm{M}_{\odot} \mathrm{yr}^{-1}$. It is remarkable that there is no significant difference in $\dot{M}$ between class 0 and I sources although our sample sources are still limited. This is inconsistent with the result by Bontemps et al. (1996) that class 0 sources show higher $\dot{M}$ than class I sources (see also Henriksen et al. 1997). This inconsistency may result from differences in methods for estimating $\dot{M}$ : Bontemps et al. obtained $\dot{M}$ indirectly from the outflow power of each source, while we estimated $\dot{M}$ directly from observed parameters of $M, R$, and $V_{\text {infall }}$ (see Table 3). Direct measurements of $\dot{M}$ for more infalling envelopes are definitely important to investigate if class 0 sources really show higher $\dot{M}$.

Since $V_{\text {infall }}$ and $V_{\text {rot }}$ increase as $R^{-0.5}$ and $R^{-1}$, respectively, as infalling matter flows toward the central star (Momose et al. 1998), $V_{\text {rot }}$ will become comparable to $V_{\text {infall }}$ close to the central star. It is most likely that the infalling matter will form a centrifugally supported Keplerian disk at $R \sim R_{\mathrm{c}}$ where 
$V_{\text {infall }}=V_{\text {rot }}$. Table 2 clearly shows that every infalling envelope has a similar $R_{\mathrm{c}}$, $\sim 100 \mathrm{AU}$. Interestingly, the derived values are also very similar to sizes of compact dust disks, which are most probably Keplerian disks, around T Tauri stars (Adams et al. 1988; Lay et al. 1994; Mundy et al. 1996). These coincidences suggest that there may be some mechanism which determines the typical size of the Keplerian disks.

Acknowledgments. The BIMA array is operated by the University of California at Berkeley, the University of Illinois, and the University of Maryland, with support from the National Science Foundation. The observations presented here were carried out under collaborative works with M. Hayashi, M. Momose, P. T. P. Ho, S.-W. Lee, D. J. Wilner, N. Hirano, M. Tamura, A. I. Sargent, H. Maezawa, and S. Yamamoto.

\section{References}

Adams, F.C., Shu, F.H., \& Lada, C.J. 1988, ApJ, 326, 865

André, P., Ward-Thompson, D., \& Motte, F. 1996, A\&A, 314, 625

Beichman, C.A., Myers, P.C., Emerson, J.P., Harris, S., Mathieu, R., Benson, P.J., \& Jennings, R.E. 1986, ApJ, 307, 337

Bergin, E.A. \& Langer, W.D. 1997, ApJ, 486, 316

Bontemps, S., André, P., Terebey, S., \& Cabrit, S. 1996, A\&A, 311, 858

Chen, H., Myers, P.C., Ladd, E.F., \& Wood, D.O. 1995, ApJ, 445, 377

Fuller, G.A. \& Myers P.C. 1992, ApJ, 384, 523

Goodman, A.A., Barranco, J.A., Wilner, D.J., \& Heyer, M.H. 1998, ApJ, 504, 223

Hayashi, M., Ohashi, N., \& Miyama, S.M. 1993, ApJ, 418, L71

Henriksen, R.N., André, P., \& Bontemps, S. 1997, A\&A, 323, 549

Hirahara, Y., Suzuki, H., Yamamoto, S., Kawaguchi, K., Kaifu, N., Ohishi, M.,

Takano, S., Ishikawa, S., \& Masuda, A. 1992, ApJ, 394, 539

Kuiper, T.B.H., Langer, W.D., \& Velusamy, T. 1996, ApJ, 468, 761

Langer, W.D., Velusamy, T., Kuiper, T.B.H., Levin, S., Olsen, E., \& Migenes, V. 1995, ApJ, 453, L293

Lay, O.P., Carlstrom, J.E., Hills, R.E., \& Phillips, T.G. 1994, ApJ, 434, L75

Momose, M. 1998, private communication

Momose, M., Ohashi, N., Kawabe, R., Nakano, T., \& Hayashi, M. 1998, ApJ, 504,314

Moriarty-Schieven, G.H., et al. 2000, in preparation

Mundy, L.G., et al. 1996, ApJ, 464, L169

Ohashi, N. 1999, in Star Formation 1999, ed. T. Nakamoto, in press

Ohashi, N., Hayashi, M., Ho, P.T.P., \& Momose, M. 1997a, ApJ, 475, 211

Ohashi, N., Hayashi, M., Ho, P.T.P., Momose, M., Tamura, M., Hirano, N., \& Sargent, A.I. 1997b, ApJ, 488, 317

Ohashi, N., Lee, S.-W., Wilner, D.J., \& Hayashi, M. 1999, ApJ, 518, L41

Ohashi, N., et al. 2000, in preparation

Shu, F.H., Adams, F.C., \& Lizano, S. 1987, ARA\&A, 25, 23

Suzuki, H., Yamamoto, S., Ohishi, M., Kaifu, N., Ishikawa, S., Hirahara, Y., \& Takano, S. 1992, ApJ, 392, 551

Tafalla, M., Mardones, D., Myers, P.C., Caselli, P., Bachiller, R., \& Benson, P.J. 1998, ApJ, 504, 900

Ward-Thompson, D., Motte, F., \& André, P. 1999, MNRAS, 305, 143 
Ward-Thompson, D., Scott, P.F., Hills, R.E., \& André, P. 1994, MNRAS, 268, 276 Willacy, K., Langer, W.D., \& Velusamy, T. 1998, ApJ, 507, L171

Williams, J.P., Myers, P.C., Wilner, D.J., \& Di Francesco, J. 1999, ApJ, 13, L61

\section{Discussion}

A. Richards: Do you have ideas on the origin of clumps? If there are differences in chemical abundances, would that also affect cooling rates locally so that they would also be differentiated by density?

$N$. Ohashi: We do not have many ideas on the origin of clumps, but one possible origin would be gravitational fragmentation. We think that the clumpy structure is not due to spatial differences in chemical abundances, but is due to differences in density.

T. Velusamy: The CCS emission in TMC 1 shows a very clumpy structure. These clumps appear to trace chemical inhomogeneities rather than density structure. These clumps also show significant chemical differentiation even among the carbon chain molecules.

$N$. Ohashi: In order for CCS to deplete, there seems to be a spatial difference of temperature or density.

D. A. Williams: Are the clumps you detect in CCS gravitationally bound?

$N$. Ohashi: We do not know if the clumps are gravitationally bound. A problem is that we cannot estimate masses of clumps from our CCS data because we do not know the fractional abundance of CCS exactly.

P. Caselli: You showed that CCS seems to avoid the dust peak in cloud cores whereas $\mathrm{N}_{2} \mathrm{H}^{+}$emission well traces the dust. However, you also showed that $\mathrm{N}_{2} \mathrm{H}^{+}$is clumpy. How are these clumps located with respect to the dust peak?

$N$. Ohashi: Some of $\mathrm{N}_{2} \mathrm{H}^{+}$clumps were not detected at the position of the dust peak, whereas one $\mathrm{N}_{2} \mathrm{H}^{+}$clumps coincides with the dust peak position. Since our integrated intensity map of $\mathrm{N}_{2} \mathrm{H}^{+}$shows a peak at the dust peak position, most of the emission was detected toward the dust peak.

E. F. van Dishoeck: I was impressed by your beautiful CCS BIMA observations of pre-stellar cores. However, interferometer images can be affected by resolving out extended structure. Can you comment on how your maps were constructed and whether you corrected for missing short spacings?

$N$. Ohashi: We did not add any short spacing data when we constructed our CCS maps, which might mean our CCS maps could be severely affected by resolving out extended structures. However, the field of view of our CCS maps is $\sim 5^{\prime}$, almost the same as the sizes of the pre-stellar cores we observed. So, we think that resolving out extended structures is not a severe problem for our CCS maps. In fact our CCS map of L1498 shows a very similar structure to the map by Velusamy et al. who used both a single dish-telescope and an interferometer to make the map. 